\title{
Stability of some functional equations defined by quasiarithmetic means
}

\author{
VASILE POP
}

\section{ABSTRACT.}

We obtain results on Hyers-Ulam stability for some functional equations defined by quasiarithmetic means.

DEPARTMENT OF MATHEMATICS

TECHNICAL UNIVERSITY OF CLUJ-NAPOCA

MEMORANDUMULUI 28

400114, CLUJ-NAPOCA, ROMANIA

E-mail address: vasile.pop@math.utcluj.ro 